\title{
First Reported Year
}

National Cancer Institute

\section{Source}

National Cancer Institute. First Reported Year. NCI Thesaurus. Code C159699.

The year that something was first noted. 